\title{
Self-sustained clusters and ergodicity breaking in spin models
}

\author{
Chi Ho Yeung and David Saad \\ Nonlinearity and Complexity Research Group, Aston University, Birmingham B4 7ET, United Kingdom \\ (Received 24 April 2013; revised manuscript received 26 June 2013; published 23 September 2013)
}

\begin{abstract}
Self-sustained spin clusters are analytically linked to ergodicity breaking in fully connected Ising and Sherrington-Kirkpatick (SK) models, relating the less understood spin space to the well understood state space. This correspondence is established through the absence of clusters in the paramagnetic phase, the presence of one dominant cluster in the Ising ferromagnet, and the formation of nontrivial clusters in SK spin glass. Yet unobserved phenomena are also revealed such as a first order phase transition in cluster sizes in the SK ferromagnet. The method could be adapted to investigate other spin models.
\end{abstract}

DOI: 10.1103/PhysRevE.88.032132

PACS number(s): 05.20.-y, 75.10.Nr, 89.20.-a

Spin glasses are magnetic materials characterized by extremely slow magnetization relaxation in the absence of external field $[1,2]$. Several models have been developed to explain their behavior $[3,4]$ which in turn have revealed a rich physical picture of a rugged free energy landscape [2,5]. Remarkably, the physics of spin glasses has a nontrivial connection to interdisciplinary applications including image processing, error correcting codes, neural networks, and combinatorial optimization [6,7]. Its connection to structural glasses and supercooled liquids have also been explored to explain the physics below the glassy temperature [8,9].

Among the various spin glass models, the SherringtonKirkpatrick (SK) model [4] is arguably the most studied. One of the most intriguing features of large-scale disordered systems in general and the SK model in particular is the breaking of ergodicity where only part of state space can be explored even after a very long time (with respect to the system dimensionality); this occurs in some parameter regimes (e.g., temperature, strength of interactions, or topology), particularly in the spin-glass phase where it manifests itself through a complex symmetry structure of order parameters that describe macroscopically the corresponding state space. Although macroscopic properties of the SK model are relatively clear its microscopic features are less understood [10], in particular the existence of stable domains that are independent of the remainder of the system; these are important for gaining insight into the mechanism that gives rise to ergodicity breaking and the physical picture of spin glasses.

In this paper, we examine analytically the existence, nature, and size distribution of self-sustained clusters in the real spin space of fully connected Ising and SK models to explain ergodicity breaking, observed in the macroscopic representation of state space. We remark that a similar behavior, termed backbone or frozen variables in sparse systems [11,12], is induced by topological disorder and is therefore different from the self-sustained clusters studied here; nevertheless, sparse topologies could be analyzed by extending the method presented here.

\section{MODELS}

The SK model comprises $N$ spin variables, any two of which $i$ and $j$ interact via a ferromagnetic $\left(J_{i j}>0\right)$ or antiferromagnetic $\left(J_{i j}<0\right)$ symmetric coupling $\left(J_{i j}=J_{j i}\right)$. Coupling variables are randomly drawn from a Gaussian distribution of mean $J_{0} / N$ and variance $J^{2} / N$; our results were obtained with $J=1$. The corresponding Hamiltonian is given by $\mathcal{H}_{\mathrm{SK}}=-\sum_{(i j)} J_{i j} s_{i} s_{j}$, which sums over all unordered spin pairs $(i \neq j)$. The infinite-range Ising model (also known as the Curie-Weiss model) is a special case of the SK model with $J=0$ or $J_{0}$, and the corresponding Hamiltonian is $\mathcal{H}_{\text {Ising }}=-J_{0} \sum_{(i j)} s_{i} s_{j} / N$.

To compute physical quantities of interest, one uses the identity $\ln \mathcal{Z}=\lim _{n \rightarrow 0}\left(\mathcal{Z}^{n}-1\right) / n$ to carry out an averages over quenched variables, replacing the average of $\ln \mathcal{Z}$ by that of the replicated partition function $\mathcal{Z}^{n}$ [5]. As $N \rightarrow \infty$, solutions are described by the magnetization and inter-replica spin correlation order parameters

$$
m_{\alpha}=\frac{1}{N} \sum_{i} s_{i \alpha}, \quad q_{\alpha \beta}=\frac{1}{N} \sum_{i} s_{i \alpha} s_{i \beta},
$$

where $\alpha, \beta=1, \ldots, n$ are replica indices. In the replicasymmetric (RS) ansatz, one substitutes $m_{\alpha}=m$ for all $\alpha$ and $q_{\alpha \beta}=q$ for all $\alpha \neq \beta$. One also identifies paramagnetic ( $m=q=0)$, ferromagnetic $(m \neq 0, q>0)$ and spin glass phases $(m=0, q>0)$ as shown in Fig. 1 .

\section{SELF-SUSTAINED CLUSTERS}

Denote a set $\mathcal{C}$ of spin variables; for each spin $i \in \mathcal{C}$ we define in-cluster and out-cluster magnetic fields $u_{i}=$ $\sum_{j \in \mathcal{C}} J_{i j} s_{j}$ and $v_{i}=\sum_{j \notin \mathcal{C}} J_{i j} s_{j}$ induced by spins $i n$ and $o u t$ of $\mathcal{C}$, respectively. The total magnetic field experienced by spin $i$ is $h_{i}=u_{i}+v_{i}$. The set $\mathcal{C}$ is self-sustained if

$$
\left|u_{i}\right|>\left|v_{i}\right|, \quad \forall i \in \mathcal{C} .
$$

In other words, the magnetic field experienced by each individual spin $i$ in $\mathcal{C}$ is dominated by the contributions of peer spin variables in $\mathcal{C}$. We remark that our framework can accommodate other cluster definitions.

To obtain the distribution of clusters, we denote $\Omega(r)$ to be the number of self-sustained clusters of normalized size $r=|\mathcal{C}| / N$, and define the entropy of clusters to be $S(r)=$ $[\ln \Omega(r)] / N$. For instance, one can easily compute $S(r)$ of the Ising model at zero temperature $T=0$ where all spins are aligned. Since the couplings are uniform, Eq. (2) is satisfied for a set $\mathcal{C}$ if $r>0.5$, i.e., any grouping with at least half of the spins is self-sustained. Thus, $\Omega(r)=C_{N r}^{N}=N ! /[(r N) !(N-$ $r N)$ !] and $S(r)=-r \ln r-(1-r) \ln (1-r)$ for $r>0.5$; and 


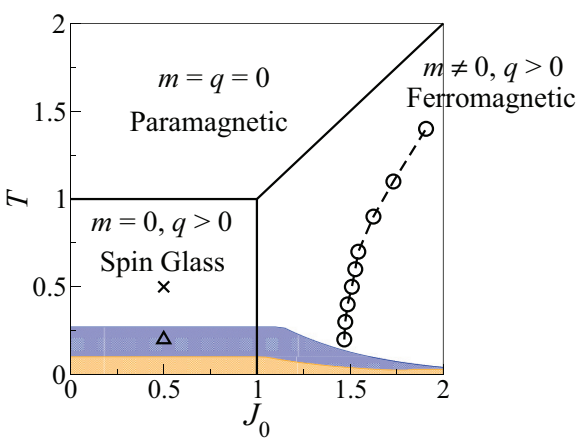

FIG. 1. (Color online) Phase diagram of the SK model as a function of coupling mean $J_{0}$ and temperature $T$. The right-hand side of the dashed line corresponds to the region where a first order phase transition in cluster sizes is observed; i.e., self-sustained clusters of certain size are absent. The blue and orange regions correspond to the negative entropy region of the RS and 1RSB ansatz, respectively; the cross and triangle symbols correspond to the points at which cluster statistics are obtained in Fig. 3(a).

$\Omega(r)=0$ and $S(r)=-\infty$ otherwise. We note that using this definition, self-sustained clusters which are subsets of larger self-sustained clusters are also counted.

We further define a variable $\sigma_{i}=1,-1$ to identify cases when spin $i$ is included in or excluded from the cluster, respectively. Consequentially, the cluster size $r=\sum_{i}(1+$ $\left.\sigma_{i}\right) / 2$. One can then define an indicator function

$$
w\left(\left\{\sigma_{i}\right\},\left\{s_{i}\right\},\left\{J_{i j}\right\}\right)=\prod_{i}\left[\frac{1-\sigma_{i}}{2}+\frac{1+\sigma_{i}}{2} \Theta\left(u_{i}^{2}-v_{i}^{2}\right)\right],
$$

where the step function $\Theta(x)=0,1$ for the cases $x<0$ and $x>0$, respectively. It turns out that the value of $\Theta(0)$ is crucial in the paramagnetic phase as will be discussed later. Thus, $w=$ 1 if the cluster defined by the set $\left\{\sigma_{i}=1\right\}$ is self-sustained, and $w=0$ otherwise.

\section{ISING MODEL}

To derive $S(r)$ for the fully connected Ising model at any temperature $T$ we uniformly sample spin configurations of given magnetization $m=\sum_{i} s_{i} / N$, as it uniquely defines the model's macroscopic properties. It is sufficient to introduce an operator partition function which measures the entropy $S(r)$ of clusters given $m$ :

$$
\begin{aligned}
& \mathcal{Z}_{\text {Ising }}(\gamma, m) \\
& \quad=\operatorname{Tr} \operatorname{Tr} w\left(\left\{\sigma_{i}\right\},\left\{s_{i}\right\}\right) \delta\left(\frac{\sum_{i} s_{i}}{N}-m\right) e^{\gamma \frac{\sum_{i}\left(1+\sigma_{i}\right)}{2}},
\end{aligned}
$$

where the dependence of $w$ on $\left\{J_{i j}\right\}$ is omitted as they are all identical $\left(J_{0}\right)$. The parameter $\gamma$ plays the role of pesudotemperature conjugate to the cluster size $\sum_{i}\left(1+\sigma_{i}\right) / 2$; by computing $\mathcal{Z}$, one obtains the entropy $S(\gamma)$ and cluster size $r(\gamma)$ as a function of $\gamma$ leading to $S(r)$.

See Ref. [13] for details of the calculation; here we briefly describe the solution. In the limit of $N \rightarrow \infty, \mathcal{Z}_{\text {Ising }}$ is given by

$$
\mathcal{Z}_{\text {Ising }}(\gamma, m)=A(m)\left[1+\Theta\left(m_{s \sigma} m\right) e^{\gamma}\right]^{N},
$$

where the prefactor $A(m)=e^{-\beta N J_{0} m^{2}}\left[2 \cosh \left(\beta J_{0} m\right)\right]^{N}$ is the entropic contribution of spin configurations $\operatorname{Tr}_{\left\{s_{i}\right\}} \delta\left(\sum_{i} s_{i} / N-m\right)$; the variable $m_{s \sigma}=\sum_{i} s_{i} \sigma_{i} / N$ and its value is given self-consistently by the equation

$$
m_{s \sigma}=m\left[\frac{\Theta\left(m_{s \sigma} m\right) e^{\gamma}-1}{\Theta\left(m_{s \sigma} m\right) e^{\gamma}+1}\right] .
$$

Using Eq. (5), one can drive the cluster size $r(\gamma)$ by

$$
r_{\text {Ising }}(\gamma, m)=\frac{1}{N} \frac{\partial \ln \mathcal{Z}_{\text {Ising }}}{\partial \gamma}=\frac{\Theta\left(m_{s \sigma} m\right) e^{\gamma}}{\Theta\left(m_{s \sigma} m\right) e^{\gamma}+1} .
$$

To compute the entropy $S(\gamma)$, one subtracts the entropic contribution $\ln A$ from $\ln \mathcal{Z}_{\text {Ising }}$ and apply the Legendre transformation to obtain

$$
\begin{aligned}
S_{\text {Ising }}(\gamma, m) & =\frac{1}{N}\left(-\gamma \frac{\partial \ln \mathcal{Z}_{\text {Ising }}}{\partial \gamma}+\ln \mathcal{Z}_{\text {Ising }}-\ln A\right) \\
& =-\frac{\gamma \Theta\left(m_{s \sigma} m\right) e^{\gamma}}{\Theta\left(m_{s \sigma} m\right) e^{\gamma}+1}+\ln \left[1+\Theta\left(m_{s \sigma} m\right) e^{\gamma}\right] .
\end{aligned}
$$

To obtain $S(r)$, we assume $m>0$ and solve Eq. (6) to obtain $m_{s \sigma}=-m$ for $\gamma<0, m_{s \sigma}=m\left(\frac{e^{\gamma}-1}{e^{\gamma}+1}\right)$ for $\gamma \geqslant 0$ and no solution in the range $-m<m_{s \sigma}<0$; as a result

$$
S(r)= \begin{cases}0 & r=0 \\ -\infty & 0<r<0.5, \\ -r \ln r-(1-r) \ln (1-r) & r \geqslant 0.5\end{cases}
$$

shown by the black line in Fig. 2. This result is valid for the entire ferromagnetic phase $(m \neq 0)$ and is consistent with $S(r)$ at $T=0$ obtained by simple counting. It implies that in the ferromagnetic phase, regardless of $T$ and $m$, clusters that include at least half of the spins are self-sustained and the magnetization is uniform over any subset of spins even for small $m$ values. Alternatively, one calculates the in-cluster and out-cluster magnetization,

$$
\left\langle s_{i}\right\rangle_{\sigma_{i}=1}=\frac{m+m_{s \sigma}}{2 r}, \quad\left\langle s_{i}\right\rangle_{\sigma_{i}=-1}=\frac{m-m_{s \sigma}}{2(1-r)},
$$

respectively, to show [using Eqs. (6) and (7)] that self-sustained clusters have the same magnetization as the out-cluster spins, $\left\langle s_{i}\right\rangle_{\sigma_{i}=1}=\left\langle s_{i}\right\rangle_{\sigma_{i}=-1}=m$.

For the paramagnetic phase, $S(r)$ is ambiguous since $m=0$ and $\left|u_{i}\right|=\left|v_{i}\right|=0$ in Eq. (2); it thus depends on the definition

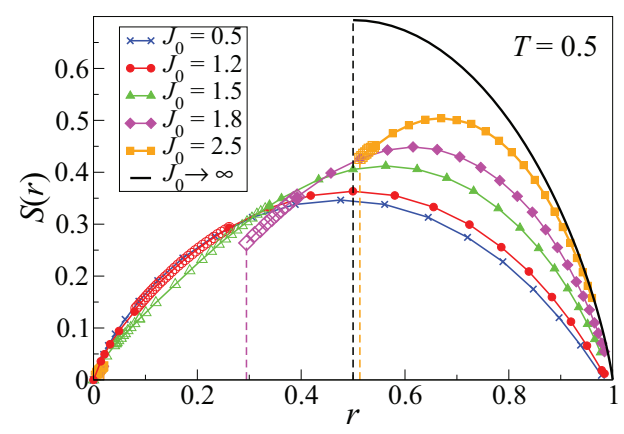

FIG. 2. (Color online) The entropy $S(r)$ of self-sustained clusters of size $r$ at $T=0.5$ and various coupling mean values $J_{0}$ in the SK model. Curves with open symbols have been obtained with a fine resolution of $\gamma$ at intervals of 0.02 . The black line $J_{0} \rightarrow \infty$ corresponds to $S(r)$ in an Ising ferromagnet. 
of $\Theta(0)$ in Eqs. (7) and (8). The choice $\Theta(0)=1$ results in $S(r)=-r \ln r-(1-r) \ln (1-r)$ for all cluster sizes $0 \leqslant$ $r \leqslant 1$, implying that any subset of spins is considered selfsustained, while for $\Theta(0)=0$ such that inequality (2) strictly holds, $S(0)=0$ and $S(r)=-\infty, \forall r \neq 0$, implying that no extensive $[O(N)]$ self-sustained clusters exist. These results suggest a correspondence between self-sustained clusters and ergodicity breaking in the Ising model, since (1) in the ergodic (paramagnetic) phase, self-sustained clusters are absent and (2) in the ferromagnetic phase with a trivial breaking of ergodicity [14], due to symmetry breaking, into regions with either $m>0$ or $m<0$, trivial clusters which span the whole system emerge.

\section{SK MODEL}

Similarly, in the SK model we uniformly draw system configurations from a distribution defined by the order parameters $\left\{m_{\alpha}\right\}$ and $\left\{q_{\alpha \beta}\right\}$, and introduce an operator partition function which measures $S(r)$ given $\left\{m_{\alpha}\right\}$ and $\left\{q_{\alpha \beta}\right\}$. Unlike the Ising model with a single order parameter $m$, the order parameters in the SK model are labeled by replica indices, and we thus define a replicated operator partition function

$$
\begin{aligned}
& \Xi_{\mathrm{SK}}\left(\gamma,\left\{m_{\alpha}\right\},\left\{q_{\alpha \beta}\right\}, n\right) \\
& =\operatorname{Tr}_{\left\{J_{i j}\right\}\left\{s_{i \alpha}\right\}} \operatorname{Tr}_{\left\{\sigma_{i \alpha}\right\}} e^{\gamma \sum_{i, \alpha} \frac{1+\sigma_{i \alpha}}{2}} \\
& \quad \times \prod_{\alpha} w\left(\left\{\sigma_{i \alpha}\right\},\left\{s_{i \alpha}\right\},\left\{J_{i j}\right\}\right) \prod_{\alpha} \delta\left(\frac{\sum_{i} s_{i \alpha}}{N}-m_{\alpha}\right) \\
& \quad \times \prod_{\alpha \beta} \delta\left(\frac{\sum_{i} s_{i \alpha} s_{i \beta}}{N}-q_{\alpha \beta}\right) \prod_{(i j)} P\left(J_{i j}\right) .
\end{aligned}
$$

We further define the corresponding unreplicated partition function with respect to spin configurations as $\mathcal{Z}_{\mathrm{SK}}\left[\gamma, P\left(m_{\alpha}\right), P\left(q_{\alpha \beta}\right)\right]$, such that $P\left(m_{\alpha}\right)$ and $P\left(q_{\alpha \beta}\right)$ are the distributions of $m_{\alpha}$ and $q_{\alpha \beta}$ in the limit $n \rightarrow 0$, and compute $\ln \mathcal{Z}_{\mathrm{SK}}=\lim _{n \rightarrow 0}\left(\Xi_{\mathrm{SK}}-1\right) / n$. To find the exact form of $P\left(m_{\alpha}\right)$ and $P\left(q_{\alpha \beta}\right)$ in the spin glass phase requires the full replica sysmetric breaking (full-RSB) ansatz, which is in principle feasible but very difficult. We will thus compute $\ln \mathcal{Z}_{\mathrm{SK}}$ under the replica sysmetric (RS) ansatz, such that $\ln \mathcal{Z}_{\mathrm{SK}}$ depends only on the variables $\gamma, m$ and $q$. We have also extended the calculation to accommodate the one-step RSB (1RSB) ansatz to compute $S(r)$ for the spin glass phase.

Even with the RS ansatz, the calculation is rather involved. We will thus describe the main rationale and results and refer readers to Ref. [13] for details. To obtain $S(r)$, we compute $r_{\mathrm{SK}}(\gamma, m, q)$ and $S_{\mathrm{SK}}(\gamma, m, q)$ by equations similar to Eqs. (7) and (8) with $\ln \mathcal{Z}_{\text {Ising }}$ replaced by $\ln \mathcal{Z}_{\text {SK }}$ and $\ln A$ replaced by the corresponding spin entropic contribution in the SK model (see Sec. S2.3 of Ref. [13]).

Figure 2 shows $S(r)$ as a function of $J_{0}$ at $T=0.5$. Remarkably, in the spin glass phase (e.g., $J_{0}=0.5$ ) cluster entropies exhibit a similar general shape to those obtained by counting in a uniform spin configuration but with degrees of freedom reduced (almost exactly) by half:

$$
S(r) \approx \frac{-r \ln r-(1-r) \ln (1-r)}{2}=\lim _{N \rightarrow \infty} \frac{\ln C_{N r / 2}^{N / 2}}{N},
$$
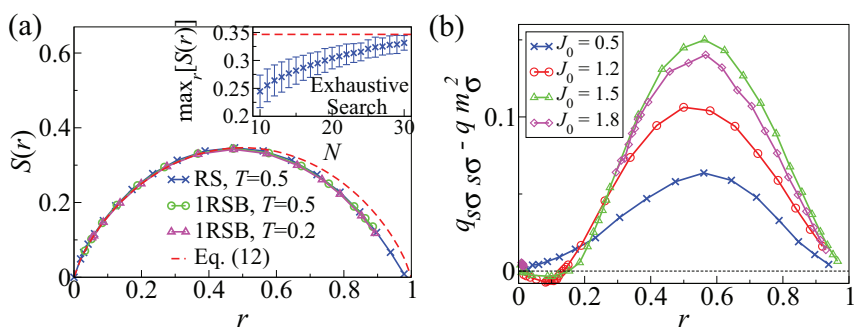

FIG. 3. (Color online) (a) Entropy $S(r)$ in the spin glass case with $J_{0}=0.5, T=0.2,0.5$, compared to Eq. (12). Inset: The maximum of $S(r)$ obtained by exhaustive search in the ground state of small SK systems with $J_{0}=0.01$, compared to the value of $S(0.5)=\left[\ln C_{N / 4}^{N / 2}\right] / N=0.346$ (red dashed line); both mean values and standard deviation are shown. (b) The difference $q_{s \sigma s \sigma}-q m_{\sigma}^{2}$ as a function of $r$ for various $J_{0}$ values.

as shown in Fig. 3(a). These results were obtained within the positive entropy region of the corresponding RS and 1RSB ansatz (see Fig. 1). We observe that this picture holds in the spin glass phase regardless of the values of $T$ and $J_{0}$. To test the validity of this result we studied numerically $S(r)$ in small SK systems of size $N=10$ to 30 by exhaustive search. The inset of Fig. 3(a) shows that $\max _{r}[S(r)]$ is approaching $\left[\ln C_{N / 4}^{N / 2}\right] / N$ as $N$ increases in agreement with the theoretical predictions.

This profile of self-sustained clusters is consistent with our understanding of the spin-glass phase: first, it shows a gap between the trivial cluster that encompasses the entire system $(r=1)$ and the exponential number of smaller self-sustained clusters which presumably correspond to suboptimal solutions; second, it shows that smaller size self-sustained clusters are determined by approximately half of their constituent spins while the other half are fixed by inherent system correlations.

To further understand the relation between self-sustained clusters and ergodicity breaking, we examine the difference $d=q_{s \sigma s \sigma}-q m_{\sigma}^{2}$ where

$$
q_{s \sigma s \sigma}=\left[\left\langle s_{i \alpha} \sigma_{i \alpha} s_{i \beta} \sigma_{i \beta}\right\rangle_{i, \alpha, \beta}\right], \quad m_{\sigma}=\left[\left\langle\sigma_{i \alpha}\right\rangle_{i, \alpha}\right],
$$

with $[\ldots]$ corresponding to the average over coupling disorders. One expects $d=0$ when the spin-configuration overlap between two replica is uncorrelated with cluster affiliations; on the other hand, $d>0$ when correlated spin configuration in two replica tend to have correlated cluster associations. Figure 3(b) shows $d$ as a function of $r$ for various $J_{0}$ values. In the spin glass phase $\left(J_{0}=0.5\right) d>0$ for all $r$, suggesting (1) the presence of self-sustained and frozen spin clusters of all size and (2) not all spin subsets constitute self-sustained clusters, or otherwise $d$ would have vanished. These results suggest that an extensive number of spin flips are required to macroscopically destabilize self-sustained clusters, which points to the existence of high-energy barriers that lead to metastable configurations. The relation to transitions observed in the state space of hard optimization problems [12] is discussed in Ref. [13]. The same phenomenon is identified as backbone or rigidity in sparse spin systems studied elsewhere. A similar picture emerges in the ferromagnetic phase with small $J_{0}>1$, except that $d \approx 0$ for small $r$ values. This result and the cluster magnetization $\left\langle s_{i}\right\rangle_{\sigma_{i}=1} \rightarrow 0$ as $r \rightarrow 0$ [see Fig. 4(b)] suggest that small self-sustained clusters are not 

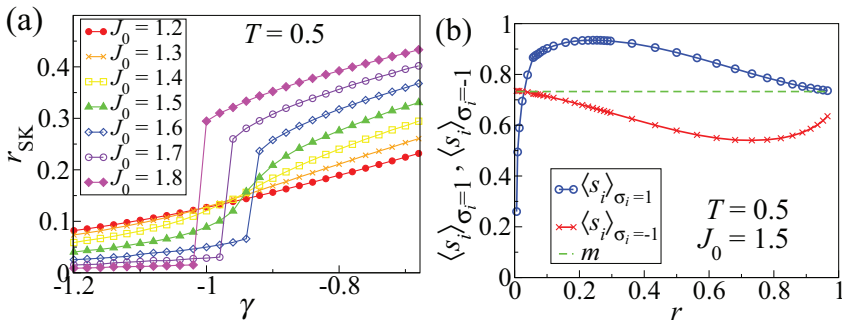

FIG. 4. (Color online) (a) The self-sustained cluster size $r_{\mathrm{SK}}$ as a function of $\gamma$ at temperature $T=0.5$ with $J_{0}$ from 1.2 to 1.8 . (b) In-cluster and out-cluster magnetizations as a function of the cluster size $r$.

frozen and thus can be easily flipped to merge into larger clusters. All these indicate that ergodicity breaking is most prominent in the spin glass phase.

We continue to examine $S(r)$ by increasing $J_{0}$, exiting the spin glass to the ferromagnetic phase, where one expects a different profile of $S(r)$ than that of Eq. (12); this difference is particularly emphasized when one considers the limit of $J_{0} \rightarrow \infty$, which corresponds to Eq. (9). The cluster entropy, shown for increasing $J_{0}$ values in Fig. 2, exhibits the onset of discontinuity in cluster sizes at $J_{0}=1.8$, implying the absence of clusters in a range of sizes. The range where discontinuity occurs increases with $J_{0}$ until $S(r)$ reduces to Eq. (9) when $J_{0} \rightarrow \infty$ as shown in Ref. [13]. To examine this behavior we plot the expected cluster size $r$ as a function of $\gamma$ in Fig. 4(a) (higher $\gamma$ selects clusters of a larger size). An abrupt jump in cluster size appears when $J_{0} \geqslant 1.6$, resembling a first order transition, which implies the emergence of large and small clusters and the absence of clusters of sizes in between. The phase boundary identifying the onset of this first order phase transition was added to the SK phase diagram in Fig. 1, denoted by open circles. This phase line marks the emergence of an extensive ferromagnetic domain which grows in size as $J_{0}$ increases and becomes the trivial cluster in the limit $J_{0} \rightarrow \infty$.

One should note that $r(\gamma)$ is not identically zero before the transition point, implying the presence of small clusters in the ferromagnetic phase, presumably small spin domains of arbitrary alignment. Figure 4(b) shows that the in-cluster magnetization $\left\langle s_{i}\right\rangle_{\sigma_{i}=1}>m$ for the entire range of $r$ except when $r \gtrsim 0$. This result and $\left\langle s_{i}\right\rangle_{\sigma_{i}=-1}<m$ suggest the presence of local domains of weaker magnetic alignment. Similar magnetization domains do not appear in the Ising ferromagnet, which suggests that coupling disorder is crucial for their formation.

In the paramagnetic phase, we have shown analytically that extensive $[O(N)]$ self-sustained clusters are absent (see Sec. S2.4 of Ref. [13]). This, in conjunction with the other results in the SK and Ising models suggest that (1) extensive self-sustained clusters are only present in nonergodic phases (e.g. ferromagnetic and spin glass phases) and (2) the cluster entropy $S(r)$ reflects the nature of ergodicity breaking: trivial ergodicity breaking due to symmetry (e.g., Ising ferromagnet) is characterized by the occurrence of trivial clusters; nontrivial ergodicity breaking (e.g., SK spin glass) is characterized by a non-trivial $S(r)$, i.e., clusters at various sizes.

\section{SUMMARY}

We showed that self-sustained clusters relate to the formation of metastable configurations separated by an extensive number of variables, which leads to ergodicity breaking. We reveal the existence of such clusters in nonergodic phases (i.e., Ising ferromagnet, SK ferromagnet and spin glass) and their absence in ergodic phases (i.e., Ising and SK paramagnet). Here we aim to establish analytically a relation between the macroscopic state space to the less understood real spin space. Other observations include a first order phase transition in the size of self-sustained clusters and the presence of domains of stronger magnetic alignment in the SK ferromagnetic regime. The new framework and understanding may also play an important role in interdisciplinary applications, particularly the development of optimization algorithms.

\section{ACKNOWLEDGMENTS}

This work is supported by the EU project STAMINA (FP7265496) and Royal Society Grant IE110151.
[1] P. Nordblad, L. Lundgren, and L. Sandlund, J. Magn. Magn. Mater. 54, 185 (1986).

[2] K. Binder and A. P. Young, Rev. Mod. Phys. 58, 801 (1986).

[3] S. F. Edwards and P. W. Andersen, J. Phys. F 5, 965 (1975).

[4] D. Sherrington and S. Kirkpatrick, Phys. Rev. Lett. 35, 1792 (1975).

[5] M. Mézard, G. Parisi, and M. A. Virasoro, Spin Glass Theory and Beyond (World Scientific, Singapore, 1987).

[6] H. Nishimori, Statistical Physics of Spin Glasses and Information Processing (Oxford University Press, Oxford, 2001).

[7] M. Opper and D. Saad, Advanced Mean Field Methods: Theory and Practice (MIT Press, Cambridge, MA, 2001).
[8] T. R. Kirkpatrick and D. Thirumalai, Phys. Rev. B 36, 5388 (1987).

[9] S. Karmakara and G. Parisi, Proc. Natl. Acad. Sci. USA 110, 2752 (2013).

[10] R. G. Palmer and C. M. Pond, J. Phys. F 9, 1451 (1979).

[11] H. Zhou, Phys. Rev. Lett. 94, 217203 (2005).

[12] L. Zdeborová and F. Krzakala, Phys. Rev. E 76, 031131 (2007).

[13] See Supplemental Material at http://link.aps.org/supplemental/ 10.1103/PhysRevE.88.032132 for details of the calculation.

[14] V. Dotsenko, Introduction to the Replica Theory of Disordered Statistical Systems (Cambridge University Press, Cambridge, 2001). 